\title{
Anabases
}

ANABASES Traditions et réceptions de l'Antiquité

29 | 2019

Varia

\section{Rétablir la métrique de Térence au XVI ${ }^{\mathrm{e}}$ siècle : le cas du Iudicium de Glaréan (1540)}

\section{Kevin Bovier}

\section{OpenEdition}

\section{Journals}

Édition électronique

URL : https://journals.openedition.org/anabases/8630

DOI : 10.4000/anabases.8630

ISSN : 2256-9421

\section{Éditeur}

E.R.A.S.M.E.

\section{Édition imprimée}

Date de publication : 14 avril 2019

Pagination : 93-105

ISSN : 1774-4296

\section{Référence électronique}

Kevin Bovier, «Rétablir la métrique de Térence au xvi siècle : le cas du ludicium de Glaréan (1540)», Anabases [En ligne], 29 | 2019, mis en ligne le 14 avril 2021, consulté le 06 novembre 2021. URL http://journals.openedition.org/anabases/8630 ; DOI : https://doi.org/10.4000/anabases.8630 

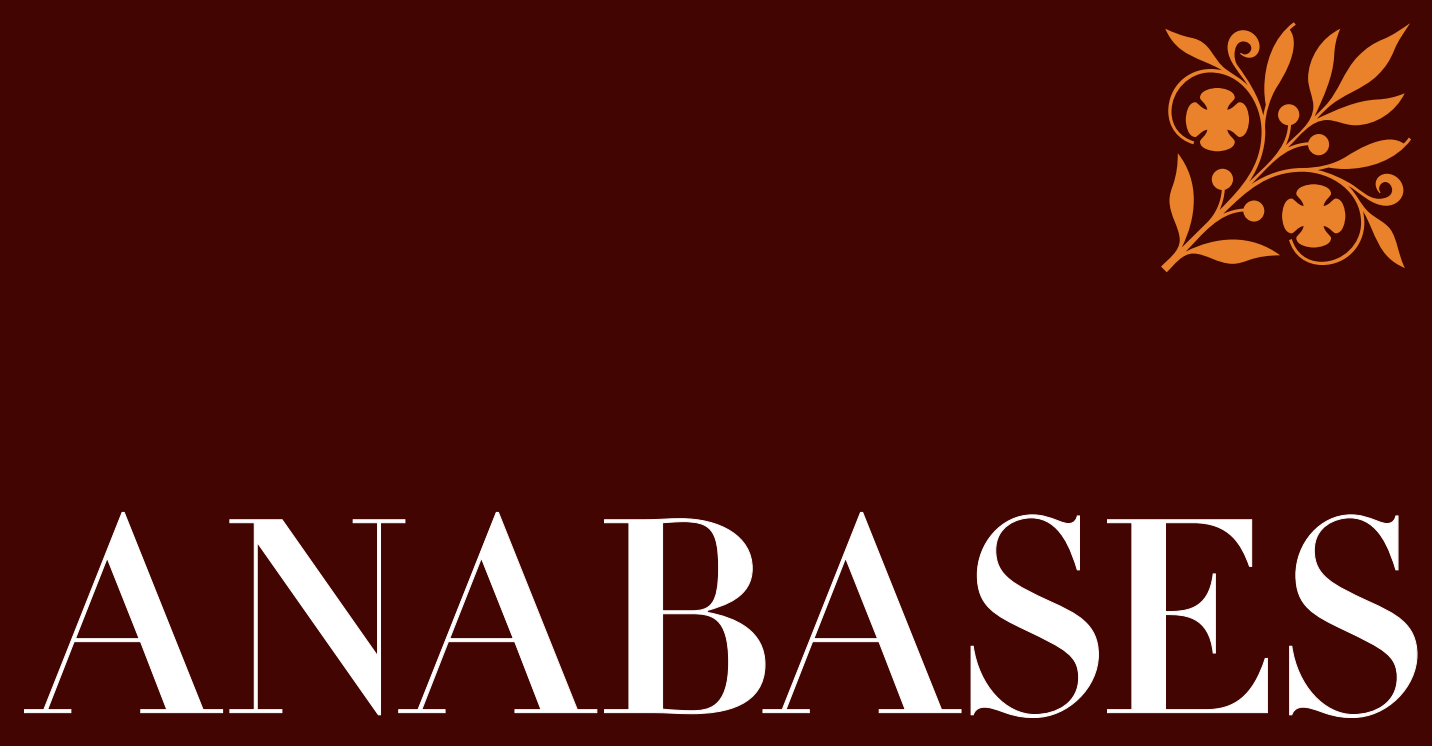

Traditions et Réceptions de l'Antiquité

\section{No29 \\ 2019}

Juliette Ernst Sculptures onctueuses de Meekyoung Shin Théâtre antique et travaux savants dans la Première modernité L'Antiquité dans la peinture (17911880) Réception d'Ovide Pierre Grimal 
ANABASES

Traditions et Réceptions de l'Antiquité

Revue de l'équipe de recherche E.R.A.S.M.E.

Université Toulouse-Jean Jaurès (UT2J)

Anabases dispose d'un Comité de lecture international. Chaque article envoyé à la rédaction est soumis, une fois anonymisé, à l'expertise de deux spécialistes qui rendent un rapport écrit. Les deux rapports anonymisés sont transmis à l'auteur qui tient compte des observations en vue de la publication.

\section{Comité SCIEnTIFIQUe}

Germaine Aujac (université Toulouse-Jean Jaurès : histoire de la géographie et des sciences antiques)

Florence Bouchet (université Toulouse-Jean Jaurès : littérature médiévale)

Hinnerk BruHns (CNRS : histoire économique et sociale ancienne et contemporaine)

Paulo Butti de Lima (université de Bari : historiographie et réception de l'Antiquité)

Luciano CANFora (université de Bari : littérature et histoire anciennes, historiographie)

Giovanna Ceserani (Stanford University : histoire intellectuelle et historiographie de la tradition classique)

Temístocles Cezar (université de Porto Alegre : historiographie moderne)

Serafina Сиомо (University of London, Birkbeck College : histoire des mathématiques et des sciences)

Paul Demont (université de Paris Sorbonne : philologie grecque et héritage classique)

Marie-Laurence Desclos (université de Grenoble II : philosophie de l'Antiquité)

Olivier Devillers (université de Bordeaux 3 - Michel-de-Montaigne : littérature et historiographie latines)

Andrea Giardina (Istituto italiano di scienze umane : histoire du monde romain et de ses réceptions)

Ève Gran-Aymerich (aibl : histoire de l'archéologie et des transferts culturels)

François HaRTog (EHEss : historiographie ancienne et moderne)

Geneviève Hoffmann (université de Picardie : histoire des mondes grecs)

Christian JACOB (CNRS/EHEss : histoire comparée et épistémologie des savoirs)

Suzanne Marchand (Louisiana State University : histoire du classicisme et de l'orientalisme)

Wilfried Nippel (Humboldt Universität Berlin : histoire et historiographie de l'Antiquité)

Sylvie Pittia (université de Paris I-Panthéon Sorbonne : histoire et historiographie du monde romain)

Stéphane Ratri (université de Franche-Comté - Besançon : philologie et héritage latin)

Comité de RÉdaction

Jacques Alexandropoulos, Marielle de Béchillon, Corinne Bonnet, Laurent Bricault, Clément Bur,

Philippe Foro, Adeline Grand-Clément, Anne-Hélène Klinger-Dollé, Véronique Krings,

Thibaud Lanfranchi, Claudine Leduc, Pascal Payen, Grégory Reimond, Catherine Valenti

Éditeur RESPonsable

Pascal PAYen

Université Toulouse-Jean Jaurès (UT2J)

SECRÉTARIAT DE RÉDACTION

Anthony Andurand / Clément Bertau-Courbières / Corinne Bonnet / Clément Bur /

Adeline Grand-Clément / Anne-Hélène Kuinger-Dollé / Véronique Krings /

Catherine Valenti (université Toulouse-Jean Jaurès) / Noémie VillacÈQue (université de Reims)

Sites Web

http://plh.univ-tlse2.fr

Revues.org : http://anabases.revues.org

Aвonnement et vente aU numéro

Éditions De Boccard - 4, rue de Lanneau - 75005 Paris

info@deboccard.com - www.deboccard.com

Tél. : 0033/(0)143260037 - Fax : 0033/(0)143548583 




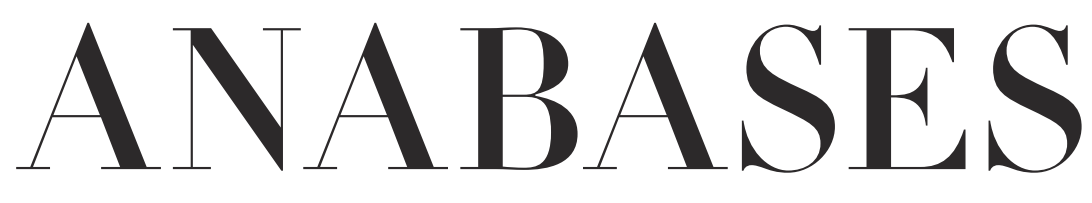

Traditions et Réceptions de l'Antiquité

$$
\begin{aligned}
& N \circ 29 \\
& 2019
\end{aligned}
$$

\section{E.R.A.S.M.E.}

Université Toulouse - Jean Jaurès 



\section{Sommaire}

$\mathrm{N}^{\circ} 29-2019$

\section{Historiographie et identités culturelles}

Ilse Hilbold

Les archives d'une bibliographe des sciences de l'Antiquité :

Juliette Ernst et la fabrique des relations internationales . . . . . . . . . I I3

Vivien LONGHI

La crise, une notion politique héritée des Grecs ? . . . . . . . . . . . 2I

Mireille Lacave-Allemand et Michel Lacave,

L’Antiquité dans la peinture en France, I79I-I880 :

une analyse quantitative à travers les Salons et les Prix de Rome . . . . . 37

Tiphaine Besnard

Du Weathering Project aux autoportraits en Venus :

Les sculptures onctueuses et savonneuses de Meekyoung Shin . . . . . 7 I

\section{Traditions du patrimoine antique}

Dossier dirigé par Pascale Paré-Rey et Malika Bastin-Hammou,

“La réception du théâtre antique dans les travaux savants de l’Europe

de la Première modernité »

Malika Bastin-Hammou et Pascale Paré-Rey

“ La réception du théâtre antique dans les travaux savants

de l'Europe de la Première modernité » . . . . . . . . . . . . . . . . 89

Kevin Bovier

Rétablir la métrique de Térence au $\mathrm{XvI}^{\mathrm{e}}$ siècle :

le cas du Iudicium de Glaréan (1540) . . . . . . . . . . . . . . . . . . . . . . 93 
Brice Denoyer

L'héritage de la métrique antique

dans l'alexandrin français au xvie siècle . . . . . . . . . . . . . IO7

Giovanna Di Martino

Vittorio Alfieri's tormented relationship with Aeschylus:

Agamennone between Tradition and Innovation . . . . . . . . . . . . . . . I2I

Marco Duranti

La condanna del prologo diegetico euripideo dagli scoli antichi

ai trattati del Cinquecento . . . . . . . . . . . . . . .

Rosario López Gregoris

L'influence de l' Arte nuevo de hacer comedias de Lope de Vega

dans l'usage des modèles classiques latins en Espagne

pendant le Siècle d'or et le Baroque » . . . . . . . . . . . . . . . . I49

Cressida Ryan

Sophoclean scholarship as a tool

to interpret eighteenth-century England . . . . . . . . . . . . . . г 6 I

Záviš ŠumaN

Axiologie critique de La Mesnardière . . . . . . . . . . . . . . . . . . . . I79

\section{Archéologie des savoirs}

Dossier dirigé par Cristina Noacco

“2000 ans déjà... Aspects de la réception d'Ovide » . . . . . . . . . . . I93

\section{La réception d'Ovide au Moyen Âge}

Jean-Marie Fritz et Cristina NoAcco

Lire Ovide au xiI ${ }^{\mathrm{e}}$ siècle : Arnoul d'Orléans

commentateur des Métamorphoses . . . . . . . . . . . . . . . $\quad{ }_{195}$

Franck Coulson

Le mythe de Pythagore dans le commentaire

“Vulgate » des Métamorphoses . . . . . . . . . . . . . . . . . . . . . . 2I

Marylène Possamaï

Comment éditer l'Ovide moralisé :

le problème de la mise en page du manuscrit Rouen Bm O.4 . . . . . . . 225

Anneliese Pollock Renck

Les Hérö̈des à la fin du Moyen Âge : pour une définition élargie de l'acte traducteur . . . . . . . . . . . . . . . . . . 239 
II. La réception d'Ovide à l'époque moderne

Fátima Díez Platas et Patricia Meilán Jácome

Le poète dans son œuvre. Ovide dans les images des Fasti

et des Tristia entre les Xv et $\mathrm{xvI}^{\mathrm{e}}$ siècles . . . . . . . . . . . . . . . . . 255

Ana Paula Rebelo Correia

Les représentations des Métamorphoses d'Ovide

dans les azulejos portugais. Influence des modèles gravés français . . . . 269

Sarah ReY

Figures d'Orphée au cinéma . . . . . . . . . . . . . . . . . . . 277

\section{Actualités et débats}

Marine LE BAIL

La modernité littéraire serait-elle affaire d'Antiquité(s) ?

Euvres \& Critiques: La contribution de l'archéologie à la genèse

de la littérature moderne, XLII, I, René Sternke dir., 20I7, 338 p. . . . . . . 2 29I

\section{Lire, relire la bibliothèque des sciences de l'Antiquité}

Éric Morvillez

“Les Horti Tauriani de Pierre Grimal

ou les prémices des Jardins romains » . . . . . . . . . . . . . . . . 30I

Pierre Grimal

“Les Horti Tauriani. Étude topographique sur la région

de la Porte Majeur ",MEFRA, tome 53, rg36. p. 25o-286 . . . . . . . . . . . 3 3i3

\section{L'atelier de l'histoire : chantiers historiographiques}

L'Antiquité au musée (coordonné par Adeline Grand-Clément) (6)

Aurélie Rodes, Catherine Valenti

Les Gaulois au musée . . . . . . . . . . . . . . . . . . . .

355

L'Atelier des doctorants (coordonné par Adeline Grand-Clément) (16)

Andrea Avalli

La question étrusque dans l'Italie fasciste $\ldots \ldots \ldots$. . . . . . . 360 
Droit et réception de l'Antiquité

(coordonné par Marielle de Béchillon et Hélène Ménard) (6)

Entre Clio et Thémis. Entretien avec Dario Mantovani, réalisé par

Hélène Ménard (Maître de Conférences d'Histoire romaine, à l'Université

Paul Valéry - Montpellier III), le 22 juin 20I8, à l'occasion de la parution

aux Belles Lettres du livre Les juristes écrivains de la Rome antique.

Les ouvres des juristes comme littérature (juin 20ı8) et de la création

de la chaire “ Droit, culture et société de la Rome antique »

au Collège de France $\left(\mathrm{I}^{\mathrm{er}}\right.$ novembre $\left.20 \mathrm{I} 8\right) \ldots$. . . . . . . . . . . . .

\section{Comptes rendus}

Philippe Borgeaud et Sara Petrella

Le singe de l'autre.

Du sauvage américain à l'histoire comparée des religions (A. Guedon) . . $\quad 37 \mathrm{I}$

Roberta Casagrande-Kim, Samuel Thrope et Raquel Ukeles (éd.)

Romance and reason. Islamic transformations of the classical past

(Cl. Bertau-Courbières) . . . . . . . . . . . . . . . . . . . . .

Hinnerk BruHns

Max Webers historische Sozialökonomie.

L'économie de Max Weber entre histoire et sociologie (Th. Lanfranchi) . . 374

Andrea Cozzo

Riso e sorriso, e altre saggi sulla nonviolenza nella Grecia antica,

(Fr. Pr. Barone) . . . . . . . . . . . . . . . . . . . 377

Franz Cumont

Manichéisme (St. Ratti) . . . . . . . . . . . . . . . 378

Emmanuelle HÉnin et Valérie NAAs (dir.)

Le mythe de l'art antique (Cl. Evrard) . . . . . . . . . . . . . . . . . 380

Jacques Jouanna, Henri Lavagne, Alain Pasquier,

Véronique SchiLtz et Michel Zink (éd.)

Au-delà du Savoir : Les Reinach et le Monde des Arts (G. Hoffmann) . . . .

382

Mario Liverani

Imagining Babylon: The Modern Story of an Ancient City (C.Bonnet) . . . 386

Françoise-Hélène Massa-Pairault, Claude Pouzadoux (Dir.)

Géants et Gigantomachie entre Orient et Occident (C.Giovénal) . . . . . . .

Scott McGill, Joseph Pucci (éd.)

Classics renewed. Reception and Innovation in the Latin Poetry

ofLate Antiquity (S. Clément-Tarantino) . . . . . . . . . . . . . . . . . . . 
Maxwell T. PAule

Canidia, Rome's First Witch (C. Landrea) . . . . . . . . . . . . . . . . . . . 39г

Jessica Priestley, Vasiliki Zali (éd.)

Brill's Companion to the Reception of Herodotus in Antiquity

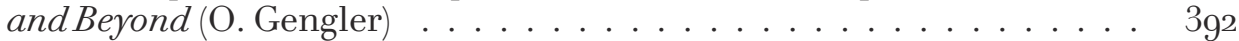

Salvatore QuAsimodo

La Lyre grecque $(\mathrm{M}$. Bianco) . . . . . . . . . . . . . . . . . . 395

Brett M. Rogers, Benjamin Eldon Stevens (éd.)

Classical Traditions in Modern Fantasy (M. Scapin) . . . . . . . . . . . . . 397

Maria Teresa Schettino et Céline UrLacher-Becht (dir.)

Ipse dixit. L'autorité intellectuelle des Anciens : affirmation,

appropriations, détournements (C. Psilakis) . . . . . . . . . . . . . 398

Guy G. Stroumsa

Religions d'Abraham : histoires croisées (D. Lorin) . . . . . . . . . . . . 400

Jean Yvonneau (éd.)

La Muse au long couteau. Critias, de la création littéraire

au terrorisme d'État (G. Hoffmann) . . . . . . . . . . . . . . 405

Résumés . . . . . . . . . . . . . . . . . . . . . 409

Index .............................. 423 



\section{Traditions}

\section{du patrimoine antique}

\section{Dossier dirigé par Pascale Paré-Rey et Malika Bastin-Hammou,}

“ La réception du théâtre antique dans les travaux savants de l'Europe de la Première modernité » 

Anabases 29 (2019), p. 93-105.

\section{Rétablir la métrique de Térence au $\mathrm{XVI}^{\mathrm{e}}$ siècle : le cas du ludicium de Glaréan (1540)}

Kevin Bovier

$\mathrm{H}$ einrich Loriti (1488-1563), dont le canton d'origine, Glaris, lui a valu le surnom latin de Glareanus, est une figure connue de l'humanisme: ses pairs saluaient à la fois ses qualités de professeur et sa polymathie. Certaines de ses œuvres ont attiré l'attention des chercheurs, notamment celles qui concernent la géographie et la musique. Les commentaires consacrés à César, Horace et Valère-Maxime ont également fait l'objet d'études. D'autres cependant sont encore à examiner, parmi lesquels son commentaire sur les comédies de Térence. L'ouvrage en question s'intitule In Publii Terentii Carmina per omneis Comoedias... Iudicium et est publié en 1540, alors que Glaréan est professeur à l'université de Freiburg im Breisgau.

Parmi les chercheurs, seuls Harold W. Lawton et Joseph A. Dane mentionnent l'ouvrage de Glaréan sur Térence. Le premier approuve un passage de l'épître dédicatoire dans lequel l'humaniste dénonce le foisonnement de commentaires inutiles sur Térence. Le second analyse un autre passage de cette épître où Glaréan évoque le travail d'Érasme sur le même auteur. En revanche, aucun d'eux n'a consulté le commentaire pour évaluer l'apport de Glaréan aux études sur Térence. C'est ce qui constituera le propos de cet article. Nous le débuterons en exposant les données d'un problème ancien que les humanistes ont tenté de résoudre : la métrique de Térence. Le commentaire de Glaréan s’inscrit en effet dans une lignée de travaux humanistes destinés à rétablir cette métrique.

Une fois les enjeux du problème identifiés, il s'agira d'examiner la manière dont Glaréan s'insère dans le débat humaniste sur la métrique : pour cela, nous analyserons successivement l'épître dédicatoire qui tient lieu de préface à l'œuvre, 
le commentaire lui-même, révélateur des méthodes de travail de Glaréan, et enfin les appendices sur la métrique que ce dernier a ajoutés en fin d'ouvrage.

\section{La transmission des comédies de Térence et le problème de la métrique}

Pour comprendre le problème de la métrique de Térence à la Renaissance, il faut revenir brièvement sur l'usage qui est fait de ses comédies au fil des siècles. Leur intégration dans le cursus scolaire dès l'Antiquité a eu pour conséquence immédiate l'apparition de commentaires qui répondaient aux besoins de l'enseignement. Parmi ces commentaires, seuls ceux de Donat et d'Eugraphius ont subsisté, auxquels il faut ajouter les Scholia Bembina contenues dans le plus ancien manuscrit des œuvres de Térence, le codex Bembinus (Vat. Lat. 3226). Térence est encore très lu au Moyen Âge, en témoignent les centaines de manuscrits conservés de cette période, sans parler des œuvres inspirées de ses pièces, comme les imitations en prose de Hrotsvita de Gandersheim au x siècle.

Le paradoxe de cette riche réception est que, dès la fin de l'Antiquité, la métrique des comédies de Térence n'est plus connue, à tel point que le grammairien Priscien, au vi ${ }^{\mathrm{e}}$ siècle, “ $[\mathbf{s}]$ 'étonne que certains nient la présence de mètres dans les comédies de Térence » (miror quosdam vel abnegare esse in Terentii comoediis metra). Dane suggère que leur utilisation comme recueil d'expressions dans un contexte scolaire a contribué à en masquer le caractère poétique. De plus, les copistes médiévaux transcrivent le texte comme de la prose et perturbent ainsi la colométrie, c'est-à-dire la disposition du texte en vers, problème qui demeure dans la plupart des incunables. La colométrie est le premier aspect que Dane distingue dans la confusion métrique chez les humanistes, les deux autres étant la prosodie (l'identification des quantités syllabiques) et la métrique (l'identification du type de mètre). L'état du texte constitue un autre point sensible du problème : sans leçons fiables, la prosodie devient aléatoire et gêne à son tour l'analyse métrique. À la période des incunables, la présence des commentaires de Donat et de Guy Jouenneaux (Guido Juvenalis) autour du texte de Térence a une influence conservatrice sur les leçons adoptées par les éditeurs. Au xvi ${ }^{\mathrm{e}}$ siècle, le déplacement de ces commentaires après le texte libère celui-ci de leur emprise.

La première difficulté, celle de la colométrie, est résolue par Ange Politien. En 1491, celui-ci reproduit à la main la colométrie et certaines variantes textuelles du codex Bembinus dans un exemplaire de l'édition vénitienne de 1475. Il faudra cependant attendre 1505 pour que Benedictus Philologus (Benedetto Riccardini) applique cette colométrie à l'édition Giunta, dans laquelle il rend hommage au travail de Politien. En revanche, les variantes que ce dernier a tirées du manuscrit n’apparaissent que sporadiquement dans le texte édité. Ce n'est qu'en 1565 que Gabriele Faerno reprend de façon systématique les leçons du Bembinus, ce qui 
constitue une étape décisive dans l'établissement du texte et la restauration de la métrique.

Néanmoins, au début du $\mathrm{xvI}^{\mathrm{e}}$ siècle, les difficultés ne sont pas toutes surmontées. Le rétablissement de la colométrie n'est que la première étape vers la compréhension de la métrique térentienne. Des paratextes sur la métrique comique apparaissent tôt dans les éditions, souvent compilés à partir des traités de grammaire antiques; mais les commentaires qui paraissent à cette période livrent peu d'éléments sur la métrique de Térence. C'est en 1532 qu'interviennent de nouvelles avancées dues à la parution de l'édition d'Érasme et du commentaire de Johann Rivius. En plus de consacrer quelques pages à la métrique comique, Érasme insère des notes succinctes dans les marges du texte, identifiant le type de mètre, signalant les élisions ou proposant des variantes textuelles. Quant aux Castigationes de Rivius, elles sont bien plus développées : il s'agit d'un commentaire lemmatique qui vise à corriger le texte et à rétablir les mètres. Mais Rivius lui-même est conscient que le travail ne fait que commencer et encourage les érudits “muni[s] d'un jugement plus pénétrant » (acriorique judicio praeditus) à le compléter et à l'améliorer. C'est peut-être en réponse à cet appel que Heinrich Glaréan publie en 1540 son Iudicium in Terentii carmina.

\section{Le ludicium de Glaréan sur la métrique de Térence (1540)}

Imprimé au format in-8, le commentaire de Glaréan paraît en 1540 chez Frellon à Lyon sans le texte de Térence. L'ouvrage débute par une épître dédicatoire datée du $1^{\text {er }}$ mars 1539 et adressée à Gérard Cizan. Le Iudicium s'étend sur deux cent quarante-trois pages. Suivant le découpage en actes et en scènes, le commentateur énumère les types de vers que lecteur va rencontrer et les difficultés générales de la scène. Il y ajoute des notes qui portent sur des problèmes plus précis, relevant de la critique textuelle et de l'identification des quantités syllabiques ou des types de pieds. Chaque note est introduite par un lemme tiré du texte, mais l'exégèse peut concerner des passages adjacents : le lemme n'est donc qu'un repère pour le lecteur qui doit avoir une édition du texte à portée de main. Le commentaire s'achève sur une brève Peroratio qui introduit les paratextes suivants : un schéma contenant les types de pieds utilisés par Térence et intitulé Communium pedum typus (fig. 1), deux pages sur les caractéristiques des vers (De carminum attributis), deux autres schémas respectivement sur les iambes (Iambicorum a monometris ad usque tetrametra generalis formula, de qua quinque ferme apud Terentium species, hic astericis notatae, reperiuntur) et les trochées (Trochaicorum eadem ratione formula tribus apud Terentium ferme speciebus, astericis pernotatis) ; enfin Glaréan revient sur les mètres iambiques dans les deux dernières pages, en concentrant son exposé sur l'évolution de ce type de mètre chez les Grecs et les Latins. 


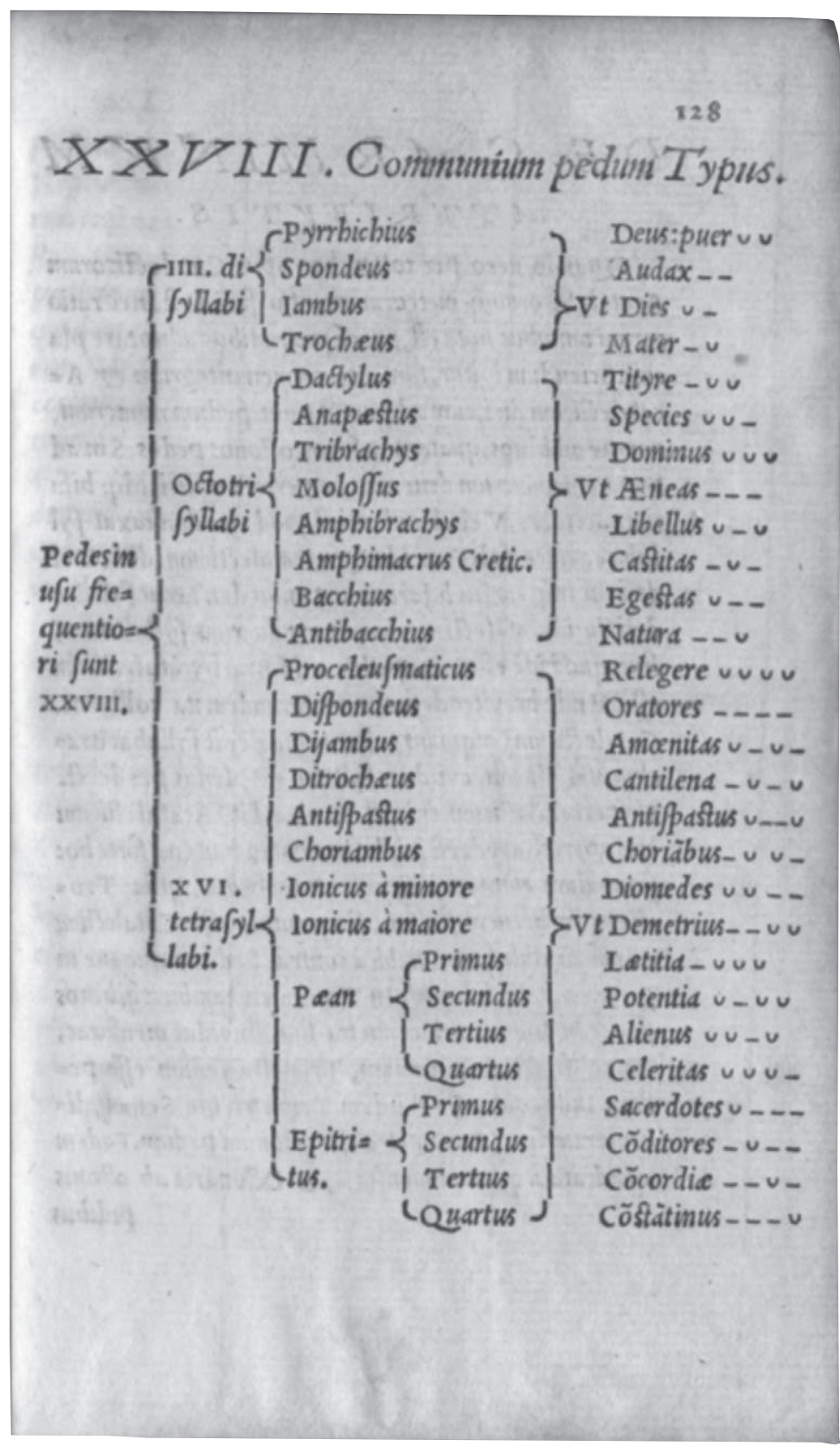

Fig. 1. Schéma des pieds utilisés par Térence dans le commentaire de Glaréan. Bayerische Staatsbibliothek München,

A.lat.a. 1867, p. 128r, urn:nbn:de:bvb:12-bsb00016459-9. 


\section{L'épître dédicatoire : entre émulation et souci d'utilité}

Dans sa lettre-préface, le Glaronnais relève le nombre important de commentaires, d'annotations et d'emblèmes qui existent déjà sur les comédies de Térence (In quem [Terentium] tot extant hodie iampridem edita commenta, tot annotationes, tot assuta emblemata ${ }^{1}$ ). Il estime cependant que peu de ces ouvrages ont une réelle valeur et dénigre en particulier celui de Guy Jouenneaux (Vt quid enim dicam de Guidone quodam Iuvenale, homine profecto ad hoc negotii inepto ?2), probablement parce qu'il n'y est question ni de critique textuelle, ni de métrique. Il valorise davantage le commentaire de Donat, bien que certains passages, à son avis, soient l'œuvre de plusieurs personnes (Donati... commentarii ipsi oppido elegantes et arguti. Verum neque unius hominis, sed saepe in unam scaenam quatuor $\left.{ }^{3}\right)$. Glaréan cite ceux qui ont entrepris de rétablir les vers avant lui : le nom de Politien apparaît, puis celui d'Érasme qui aurait travaillé sur Térence lors d'un séjour chez Aldo Manuzio à Venise, en 1507-1508 (Ante annos aliquot Politianus, ex nescio quo vetusto codice, conatus est carmina in certam redigere seriem. Post eum Erasmus praeceptor noster Venetiis apud Aldum ${ }^{4}$ ). Mais selon Glaréan, les premiers efforts d'Érasme n'auraient pas suffi : au fil du temps, le texte de Térence se serait à nouveau dégradé (non ita multo post rursus cuncta turbata sunt et intra triginta annos author ita a priore emendatione exorbitavit $\left.{ }^{5}\right)$; Érasme aurait alors repris sa tâche à un âge avancé, mais en travaillant à la hâte (Erasmus, iam extrema aetate, alteram adhibuerit manum, sed quatuor duntaxat dierum opera ${ }^{6}$. Et de fait, l'humaniste des Pays-Bas, dans son édition, reconnaît n'y avoir consacré que quatre jours (nos quatriduanam opellam sumpsimus ${ }^{7}$ ). En revanche, il ne mentionne pas son séjour chez Manuzio ${ }^{8}$ : Glaréan en a peut-être exagéré l'importance pour minimiser les progrès réalisés sur la métrique térentienne durant les décennies précédentes et pour légitimer son propre commentaire. Érasme aurait utilisé les annotations manuscrites que Glaréan avait faites dans une édition (usus ibi codice meo, in quo pleraque annotata erant $\left.{ }^{9}\right)$ : celui-ci fait donc part de sa contrariété, car, assure-t-il,

\footnotetext{
1 GLARÉAn, Iudicium, p. 2r-v.

2 Glaréan, Iudicium, p. $2 \mathrm{v}$.

3 Glaréan, Iudicium, p. 2v.

4 Glaréan, Iudicium, p. 2v.

5 Glaréan, Iudicium, p. 2v.

6 Glaréan, Iudicium, p. 2v.

7 Ed. Froben (voir n. 23), f. ${ }_{\text {a } 3}$ r.

8 DANE, “ On metrical confusion... », p. 120-121 et 125, estime que le travail d'Érasme à Venise n'a pas influencé les éditions ultérieures des comédies, pas même l'aldine de 1517.

9 Glaréan, Iudicium, p. 3r.
} 
“j’avais rédigé ces notes pour moi, non pour les autres, et certainement pas pour qu'elles fussent publiées dans cette édition "(Quam rem non admodum aequo animo tuli. Nam ego mihi ea, non aliis notaveram, nec in hoc certe ut ederentur ${ }^{10}$ ), c'est-à-dire celle qu'Érasme fit paraître chez Froben en 1532. Dans une note, Glaréan précise que l'exemplaire annoté de sa main était l'aldine de $1517^{11}$.

Piqué dans son orgueil, Glaréan est alors revenu à ces «bagatelles » (ad has nugas denuo relapsus) pour « faire part au lecteur de [s] on jugement sur l'arrangement de tous les vers qui se trouvent chez cet auteur "(de omnium quae apud hunc authorem sunt, carminum ratione meum lectori iudicium aperire $\left.{ }^{12}\right)$. Il se dit étonné que les grammairiens aient si peu écrit sur la métrique de Térence. Parmi ceux qu'il mentionne, Héphestion ${ }^{13}$, auteur d'un manuel de métrique, lui paraît le plus pertinent; c'est de ce grammairien grec qu'il s'inspire pour élaborer les schémas qui se trouvent après le commentaire. Glaréan souligne l'audace (licentia) croissante des poètes grecs et latins à l'égard du mètre iambique ; selon lui, cette caractéristique atteint son paroxysme avec la poésie de Térence, dans laquelle tout ou presque est permis. Cette explication topique apparaissait déjà chez Érasme ${ }^{14}$. Glaréan expose ensuite sa vision de la métrique comique et de son évolution, les types de pieds et les élisions. Il reconnaît néanmoins que la complexité de cette métrique “ crée beaucoup d'embarras partout »(Quae omnia multum negocii ubique facessunt nobis $\left.{ }^{15}\right)$. La lettre se conclut par l'éloge de son dédicataire et la mention de deux autres témoins de son travail acharné, Pierre Pétremand et Guillaume Barot, probablement des étudiants bourguignons qui ont résidé chez Glaréan ${ }^{16}$.

Cette préface contient quelques renseignements sur l'élaboration du commentaire : Glaréan a l'ambition de fournir un ouvrage plus utile que la plupart des commentaires existants et qui soit dans la continuation des travaux de Politien et d'Érasme ; il souhaite même dépasser l'apport de ce dernier. Cette rivalité avec son maître se retrouvera dans le commentaire. Les connaissances que Glaréan

10 GlarÉan, Iudicium, p. 3r.

11 Glaréan, Iudicium, p. 65r.

12 Glaréan, Iudicium, p. 3r.

13 Métricien alexandrin du II ${ }^{\mathrm{e}}$ siècle apr. J.-C., Héphestion fut peut-être un précepteur de l'empereur Lucius Vérus. Il est l'auteur d'une œuvre de métrique considérable dont il reste un manuel (Encheiridion). À cela s'ajoute un traité fragmentaire sur la poésie (S. Fornaro, s.v. “ Hephaistion [4] », DNP 5, 1998, col. 350-352).

14 Ed. Froben (voir n. 23), f. ${ }_{2}$ r.

15 Glaréan, Iudicium, p. 5r.

16 H. Schreiber, Heinrich Loriti Glareanus, seine Freunde und seine Zeit, Freiburg i. B., 1837, p. 105, n. 255. Leur nom n'apparaît pas dans la matricule de l'université de Freiburg (H. Mayen (hrsg.), Die Matrikel der Universität Freiburg im Breisgau, von 14601656, 2 vol., Freiburg i. B., 1907-1910). 
a acquises en matière de métrique viennent des grammairiens antiques, grâce auxquels il a pu élaborer les schémas qui seront étudiés plus loin. La difficulté de la métrique de Térence, due à la grande diversité des pieds qui la compose, contraint cependant Glaréan à des tâtonnements pour améliorer le texte et rétablir sa scansion, comme on le verra en examinant le commentaire.

\section{Le Iudicium ou le commentaire comme lieu de débat}

Il s'agit à présent de voir quelles méthodes a employées Glaréan pour corriger le texte et pour identifier les pieds dans les vers. Ces deux étapes ont exigé du commentateur l'usage de sources diverses. Tout d'abord, Glaréan ne semble pas avoir utilisé de manuscrits, malgré la terminologie parfois ambiguë à laquelle il recourt dans ses notes : les termes de codices vetusti ou encore d'antiquus codex qui s'y trouvent renvoient à des éditions anciennes des comédies de Térence. Ainsi, lorsqu'il parle de l'édition aldine de 1517 et d'une autre plus ancienne (peutêtre celle de Soardi de 1512), il écrit : Aldinus codex atque adeo vetustus alter ${ }^{17}$. Il possédait un exemplaire de chacune de ces éditions vénitiennes ${ }^{18}$; celle de Soardi comprend même des annotations de la main de l'humaniste, mais c'est celle d'Aldo Manuzio qui fournit à Glaréan le texte de base pour son commentaire. C'est en outre celle qu'il cite le plus dans ses notes. Glaréan mentionne également une édition imprimée à Cologne (codex Agrippinensis); il est difficile de l'identifier avec précision, mais elle contenait les commentaires d'Érasme et de Rivius auxquels il se réfère constamment. L'édition bâloise réalisée par Érasme est aussi indiquée sous le nom de codex Frobenii ou decodex Erasmi : elle incluait le commentaire de Giovanni Calfurnio (Johannes Calphurnius), que Glaréan cite à plusieurs reprises dans ses notes. Toutes ces éditions, et peut-être d'autres encore, lui ont permis de comparer les différentes leçons retenues par ceux qui ont travaillé sur le texte avant lui.

À la collation des éditions s'ajoute la consultation des commentaires d'Érasme et de Rivius, deux auteurs qu'il respecte, appelant le premier « notre précepteur » (praeceptor noster) et disant du second qu'il « a écrit élégamment sur cet auteur [Térence], à la fois soigneusement et abondamment »(Ioan. Rivius..., qui in hunc authorem et diligenter et oppido eleganter scripsit ${ }^{19}$ ). Glaréan tient compte de leur opinion dans ses notes avant de prendre position. Il n'hésite toutefois pas à se montrer critique envers eux s'il estime qu'ils se sont trompés. Il polémique en particulier avec Érasme qu'il connaît personnellement. Dans une note sur l'Andrienne, Glaréan rapporte qu'Érasme et lui n'étaient pas d'accord sur certains

17 GlarÉAn, Iudicium, p. 61r.

18 I. Fenlon et I. M. Groote, “ Heinrich Glarean's Books », in I. Fenlon et I. M. Groote (eds.), Heinrich Glarean's Books, Cambridge, 2013, p. 328.

19 Glaréan, Iudicium, p. 6v. 
vers de cette pièce; au cours du débat, Érasme aurait invoqué l'autorité de Priscien, Glaréan celle de Térentianus Maurus (ille Priscianum haberet authorem, ego Terentianum $\left.{ }^{20}\right)$. L'émulation entre les deux humanistes est flagrante dans la suite de cette note, où transparaît chez Glaréan un mélange d'admiration et d'agacement vis-à-vis de son mentor :

“ Tandis que j’avançais ces arguments et quelques autres pour défendre mon opinion, il [Érasme] écoutait en faisant la sourde oreille. Ainsi il traita personnellement des treize premiers vers de cette scène, afin que je prisse l'habitude d'admirer plus souvent ce qui lui venait alors à l'esprit. Mais nous voyons, par Hercule, la nature nous pousser à écouter avec gravité les hommes de plus grande réputation, plus rarement les plus jeunes, même ceux qui donnent de bons conseils, surtout lorsqu'un contentieux survient à propos d'affaires intellectuelles. C'est que la faiblesse humaine est avide de victoire. »

Haec et quaedam alia cum pro meae opinionis defensione adduxissem, ille surda aure audiebat. Itaque ipse hujus scenae XIII primos versus ita tractavit, ut saepius mecum mirari soleam, quid ei tunc venerit in mentem. Sed ita, Hercules, natura comparatum videmus, ut majoris existimationis homines, nonnunquam minores, etiam bene admonentes, gravatim audiamus, maxime ubi de ingenii negotiis contentio incidit. Adeo victoriae est cupida humana imbecillitas ${ }^{21}$.

Ailleurs toutefois, Glaréan estime que le travail d'Érasme, quoique hâtif, est supérieur à celui des autres commentateurs ${ }^{22}$. Ce débat sur le texte et la métrique de Térence précède même la parution de l'édition érasmienne de 1532 : dans une lettre de l'automne 1519, l'humaniste Beatus Rhenanus signale en effet à Wilhelm Nesen que “Glaréan a restauré de nombreux passages de Térence à propos desquels il va écrire à Érasme "(Glareanus restituit multa Terentii loca, de quibus ad Erasmum scripturus est ${ }^{23}$ ). La discussion entre érudits pouvait donc prendre différentes formes, orales ou écrites, et se retrouver plus tard dans les commentaires.

Outre les travaux d'Érasme et de Rivius, l'humaniste glaronnais a consulté le commentaire de Giovanni Calfurnio ${ }^{24}$ sur le Heautontimorumenos, car celui de

20 Glaréan, Iudicium, p. 21v.

21 Glaréan, Iudicium, p. 22r.

22 GLARÉAn, Iudicium, p. 104v.

23 A. Horawitz et K. Hartfelder (hrsg.), Briefwechsel des Beatus Rhenanus, Leipzig, 1886, p. 185. Rhenanus a lui-même annoté une édition strasbourgeoise de Térence datée de 1516 : voir à ce sujet G. Freyburger, "Beatus Rhenanus annotateur de l'Heautontimoroumenos de Térence ", in J. Hinstein (éd.), Beatus Rhenanus : lecteur et éditeur des textes anciens, Turnhout, 2000, p. 117-128.

24 Ce commentaire paraît pour la première fois à Venise, chez Jacques le Rouge (Jacobus Rubeus), en 1476. 
Donat est perdu pour cette pièce. Glaréan y cherche une confirmation ou une infirmation des leçons de l'édition. En revanche, le nom de Guy Jouenneaux, critiqué dans l'épître dédicatoire, n’apparaît pas dans les notes de Glaréan. Jouenneaux avait produit un commentaire à l'imitation de Donat ${ }^{25}$, mais son contenu ne permettait pas à Glaréan d'améliorer le texte ou sa métrique.

On a vu plus haut Érasme et Glaréan s'affronter par l'intermédiaire de Priscien et Térentianus, deux grammairiens faisant figure d'autorités dans le débat entre humanistes. Térentianus Maurus est l'auteur d'un De litteris, syllabis, metris et Priscien a composé des Institutiones grammaticae et un De metris comicis. Glaréan avait un exemplaire imprimé des œuvres du second (Venise, 1500) ${ }^{26}$. Il trouvait dans ces ouvrages des passages des comédies utilisés comme exemples. Ces passages présentaient parfois des variantes par rapport au texte des éditions, ce qui permettait ensuite au commentateur de valider ou non ses analyses métriques.

Plus ponctuellement, Glaréan mentionne d'autres travaux humanistes, comme les Elegantiae de Lorenzo Valla, dont il possédait une édition parisienne de 1520, ou encore le Cornucopiae de Nicolo Perotti, dont l'édition vénitienne de 1513 était également dans ses rayons ${ }^{27}$. Ces deux ouvrages constituent des sources habituelles pour les humanistes qui y trouvaient un grand nombre de remarques sur les œuvres antiques, principalement sur la langue. Les Adages d'Érasme figurent aussi dans les ouvrages cités : certains adages ont d'ailleurs été recopiés par Glaréan dans son édition de Térence de $1517^{28}$. Il pouvait ainsi comparer les citations de Térence retenues dans les Adages avec le texte de l'édition.

Concernant l'identification des mètres, Glaréan dénonce la confusion qui règne entre iambes et trochées et reproche à Érasme sa présentation peu claire des mètres de Térence. Selon lui, il est possible d'y mettre de l'ordre et de classer ces mètres sans aller contre l'art du poète. Pour arriver à ce résultat, Glaréan affirme reprendre de manière plus cohérente la tâche de son maître, sans inventer de nouvelles audaces (novas licentias $^{29}$ ). En affichant ainsi son objectif et sa méthode de travail, il annonce aussi les schémas métriques qui suivent le commentaire. Malgré son aplomb assumé et ses critiques à l'encontre de ses prédécesseurs, Glaréan reconnaît qu'il reste des problèmes à résoudre et en

25 J. Bloemendal, “ In the Shadow of Donatus : Observations on Terence and Some of his Early Modern Commentators ", in K. Enenkel et H. Nellen (eds.), Neo-Latin Commentaries and the Management of Knowledge in the late Middle Ages and the Early Modern Period (1400-1700), Louvain, 2013, p. 299.

26 Fenlon et Groote, “ Heinrich Glarean’s books », p. 326.

27 Fenlon et Groote, “ Heinrich Glarean's books », p. 330-331.

28 A. Horz, "A Dorio ad Phrygium: Glarean and the Adagia of Erasmus » in Heinrich Glarean's Books, p. 182-183 et n. 13.

29 GlarÉAn, Iudicium, p. 30r. 
appelle régulièrement au jugement du lecteur, signe qu'il n'est pas toujours sûr du bien-fondé de ses propositions.

En dépit des difficultés, le commentateur estime maîtriser suffisamment la métrique de Térence pour en tirer des conclusions générales : “ Toutes les comédies de Térence commencent en effet par des sénaires iambiques et finissent en tétramètres trochaïques catalectiques "(Omnes enim Terentii Comoediae ab iambicis senariis incipiunt et in trochaicos tetra[metros] catalecti [cos] finiunt $\left.{ }^{30}\right)$. Par ailleurs, Glaréan est capable d'identifier les procédés métriques couramment utilisés par Térence : « En huitième position, la première [syllabe] d'aedepol a de nouveau été rendue brève, comme souvent chez ce poète » (In octavo [loco] rursus 'aedepol' prima correpta, ut frequens est huic poetae $\left.{ }^{3 \mathbf{1}}\right)$. Le commentateur repère aussi les traits archaïques de la langue de Térence, ce qui lui permet de deviner les quantités de certaines syllabes : “Ainsi aufugistin’ et satin’, dans ce vers, sont brefs selon un usage que Térence conserve obstinément à la suite d'Ennius et de tous les autres " (Sic aufugistin' et satin' in hoc carmine sunt brevia usu, quem Terentius mordicus obtinet post Ennium ac caeteros ${ }^{\mathbf{3 2}}$ ).

Enfin, le commentaire comporte des parallèles avec les textes littéraires antiques. Il s'agit surtout de pièces poétiques d'auteurs tels que Virgile et Horace, et à quelques reprises Plaute, Juvénal, Ovide et Lucain ; la prose est citée plus rarement (Cicéron, Salluste). Glaréan effectue des rapprochements métriques et linguistiques avec ces textes. Il compare également plusieurs passages de Térence et estime que “ ce poète a pris plaisir à faire des vers ambigus » (Habuit hoc poeta pro voluptate, opinor, ut ambiguos versus faceret $\left.{ }^{33}\right)$. Si la licentia en matière de métrique est un motif de plainte régulier chez Glaréan, elle devient parfois un argument pour justifier certaines propositions du commentateur, que celui-ci juge lui-même audacieuses.

Ainsi l'utilisation des sources dans le commentaire est double: les unes amènent des variantes pour rétablir le texte, les autres contribuent plutôt à restituer la métrique. Dans la première catégorie se trouvent les éditions, que Glaréan collationne pour obtenir les variantes de la tradition, faute d'avoir accès à des manuscrits. Les commentaires peuvent receler des leçons divergentes, mais Glaréan les consulte aussi pour avoir l'avis de ses prédécesseurs sur certains passages, qu'il s'agisse de critique textuelle (conjectures) ou d'analyse métrique (identification des pieds). Certaines sources font double emploi : les traités des grammairiens ont permis à Glaréan d'acquérir les connaissances théoriques sur la métrique comique, mais dans la perspective concrète du commentaire ce sont

30 GLarÉAn, Iudicium, p. 112r.

31 Glaréan, Iudicium, p. 53v.

32 GlarÉan, Iudicium, p. 51r.

33 Glaréan, Iudicium, p. 46r. 
les exemples tirés des comédies de Térence qui lui ont été les plus utiles. Les textes littéraires antiques servent de révélateurs de la langue et de la métrique particulières de Térence. Les autres écrits humanistes fournissent à Glaréan des indications linguistiques (les Elegantiae de Valla) ou des leçons textuelles (les Adages d'Érasme). La tentative de rétablir le texte n'est pas originale, puisque Rivius avait appliqué la même méthode dans ses Castigationes. Le raisonnement philologique, qui demeure largement empirique, se heurte à la métrique souvent fluctuante de Térence: dès lors, le manque d'assurance dans les domaines textuel et métrique empêche l'exégète de proposer une solution définitive. Enfin, l'omniprésence de la rivalité avec Érasme dans le commentaire incite à penser que Glaréan voulait afficher son érudition en s'attaquant à un sujet difficile.

\section{Les schémas métriques : un mode d'emploi pour débutants}

Le commentaire est suivi d'une brève peroratio où Glaréan présente un schéma (typus) qu'il a établi à la page suivante. Il comporte les types de mètres les plus courants accompagnés d'exemples, comme Tityre pour le dactyle ou dominus pour le tribraque. Le commentateur poursuit son objectif pédagogique dans une annexe intitulée De carminum attributis. Dans ce petit texte à l'usage des débutants (pro rudibus), il revient sur les notions métriques de base. Deux schémas le complètent, l'un sur les vers iambiques, l'autre sur les vers trochaïques ; Glaréan les classe du monomètre au tétramètre en fournissant un exemple pour chaque cas. Ainsi, dans le schéma iambique, le monomètre acatalectique est représenté par Musae deae; pour les trochées, le trimètre brachycatalectique est illustré par la phrase Musa dulcis est Horatiana. Deux schémas plus brefs indiquent respectivement la formule de base du vers iambique (l'exemple donné, suis et ipsa Roma viribus ruit, est composé uniquement d'iambes) et les positions potentielles des différents pieds qu'on rencontre dans le vers iambique. Un dernier schéma montre la formule la plus libre (licentiosissima) du vers iambique, avec toutes les possibilités de substitution. Il faut insister sur l'originalité de Glaréan dans sa manière de présenter les mètres de Térence : les travaux de ses prédécesseurs contiennent certes quelques développements théoriques à ce sujet, généralement tirés des traités de grammaire antiques, mais aucun n'expose aussi clairement la métrique comique.

Cette profusion de paratextes destinés aux lecteurs « débutants » laisse penser que le commentaire lui-même ne s'adresse pas qu'à des humanistes chevronnés. Cette impression est confortée par l'avis au lecteur qui précède les notes sur la première pièce, l'Andrienne: Glaréan y réitère son intention d'être utile aux débutants (rudibus prodesse) en leur rappelant parfois quelques notions de base (rudia) dans ses notes ${ }^{34}$. 
Le format in-8 du livre est d'ailleurs adapté à un tel usage. Le commentaire est en outre construit comme un correctifà l'édition d'Érasme, qui considérait Térence comme un auteur aux intérêts multiples dans l'apprentissage humaniste ${ }^{35}$. Enfin, on a vu Glaréan mentionner des étudiants français dans la préface. Néanmoins, contrairement au commentaire sur Horace qui était surtout destiné à ses étudiants de Freiburg ${ }^{36}$, le Iudicium, imprimé à Lyon, vise une audience plus variée. En laissant fréquemment le lecteur juger des passages, Glaréan ouvre la porte au débat et s'adresse alors aux humanistes confirmés, capables de donner leur avis sur ce sujet complexe. En ce qui concerne sa fortune éditoriale, le Iudicium a été réédité une fois à Venise en $1543^{37}$ avant de se retrouver au milieu d'autres commentaires dans un grand nombre d'éditions postérieures ${ }^{38}$. Il semble avoir été imprimé pour la dernière fois à Venise en $1586^{39}$.

\section{Conclusion}

Dans l'introduction à son édition des comédies de Térence, Jules Marouzeau prétend qu'il n'y a pas de travail sur la métrique térentienne avant celui qui accompagne l'édition de Gabriele Faerno en $1565^{40}$. Ce jugement est en réalité tributaire de celui de Richard Bentley, qui fut le premier à maîtriser les principes métriques de Térence (dont il publie les œuvres en 1726) et qui reconnaissait l'importance du travail préalable de Faerno ${ }^{41}$. Cependant, comme on l'a vu, les

35 Voir Érasme, De ratione studii, in J.-Cl. Margolin (ed.), Opera omnia, I.2, Amsterdam, 1971, p. 136-138.

36 WIENER, " Glarean's didactic approach to Horace », p. 226.

37 P. Terentii Aphri Comoediae sex, summa denuo vigilantia recognitae. Item Henrici Loriti Glareani, in P. Terentii carmina per omneis eius comoedias iudicium, Venise : C. da Trino, 1543.

38 Sur la fortune des éditions et commentaires sur Térence du $\mathrm{xv}^{\mathrm{e}}$ à la fin du $\mathrm{XvI}^{\mathrm{e}}$ siècle, voir P. F. Genl, “Selling Terence in Renaissance Italy: The Marketing Power of Commentary ", in Chr. Kraus and Chr. Stray (éds.), Classical Commentaries: Explorations in a Scholarly Genre, Oxford, 2016, p. 253-274.

39 P. Terentius Afer cum commentariis Aelii Donati, Guidonis Iuvenalis Cenomani, Petri Marsi in omnes fabulas, Io. Calphurnii Brixiensis in Heautontimorumenon... Henrici Loriti in carmina Terentii per omnes eius comoedias iudicium, Venezia : G. Griffio, 1586 [USTC 858813].

40 J. Marouzeau (éd.), “Introduction », in Térence, Comédies, t. I : Andrienne, Eunuque, Paris, Les Belles Lettres, 1947, p. 53.

41 Reynolds, Wilson, Scribes and Scholars, p. 187. Voir l'avis au lecteur de Bentley dans son édition: P. Terentii Afri Comoediae. Recensuit notasque suas et Gabrielis Faerni addidit Richardus Bentleius. Editio altera denuo recensita, ac indice amplissimo rerum 
humanistes n'ont pas attendu Faerno pour aborder le problème de la métrique térentienne. Le rétablissement de celle-ci n'est pas le fait d'un seul individu plus éclairé que les autres ; c'est le résultat d'un processus de plusieurs décennies qui implique de nombreux humanistes.

Glaréan avait toutefois ses propres raisons de commenter les comédies de Térence, en particulier leur aspect métrique : il y a une préoccupation pédagogique, celle de faire progresser les connaissances sur un auteur qui occupe une place majeure dans l'éducation humaniste ; il y a aussi le défi intellectuel représenté par la métrique si particulière de Térence. Érasme a sans doute joué un rôle dans la motivation de son élève, au-delà même de l'affaire des notes de Glaréan que le maître aurait indûment utilisées. Enfin, il ne faut pas négliger le prestige que pouvait valoir à Glaréan ce commentaire en raison de la popularité de Térence au sein du mouvement humaniste.

Du point de vue méthodologique, Glaréan suit les normes du commentaire humaniste : en l'absence de manuscrit donnant un texte fiable, le commentateur recourt à toutes sortes de sources, textes littéraires, éditions et commentaires anciens, travaux divers d'humanistes, afin de rétablir le texte des comédies et, in fine, la métrique térentienne. Au milieu de ces sources se détache la figure d'Érasme : son édition a servi de base à Glaréan pour son commentaire, mais le Glaronnais s'est également souvenu des discussions qu'il avait eues dans le passé avec son maître. Le iudicium évoqué par Rivius et repris par Glaréan apparaît dès lors comme le maître-mot de ce type d'exégèse : il convient à la fois pour désigner la manière dont le commentateur utilise ses sources et pour faire valoir son opinion dans le débat humaniste.

\author{
Kevin Bovier \\ Université de Genève \\ Département des sciences \\ de l'Antiquité \\ 5 , rue De-Candolle \\ 1211 Genève 4 \\ kevin.bovier@unige.ch
}

et verborum, tam in textum quam notas, aucta, Amsterdam : R. et J. Wetstenios et G. Smith, 1727, f. [:4] . 